\title{
Design and performance
}

\section{Improving open canned systems}

\author{
The results of a study on the hydraulic performance of \\ open-bottom canned pump intakes should aid engineers and \\ pump users to design these systems with reduced velocity \\ fluctuations and undesirable flow rotation. David Sanders of \\ Sunrise Engineering with Steven L. Barfuss and Michael C. Johnson \\ of Utah State University provide the details.
} ump systems often require the
use of an open-bottom canned
intake to house the vertical

turbine pump. A canned pump can be defined as one in which the pumping unit is housed within a shaft and may be considered in two separate categories - open-bottom and closed-bottom. Open-bottom canned pumps draw water from a horizontal header, which passes flow along the bottom of the open can with enough head to at least submerge the pump bell within the can. Closed-bottom canned pumps are enclosed at the bottom, and water fills up the can by way of an inlet pipe on the side of the can. This article investigates open- bottom canned pump intakes and the measures necessary to improve hydraulic performance and reduce velocity fluctuations and flow rotation under varied conditions.

In open-bottom canned pump systems where the average velocity in the header (perpendicular to the can) becomes greater than the average velocity in the riser, a number of potential problems may arise. Of these, the potential for vortices, flow prerotation (annular flow pattern with tangential velocity occurring upstream of the turbine) to the pump impellers, turbulence, and unsteady and nonuniform flow at the pump throat can

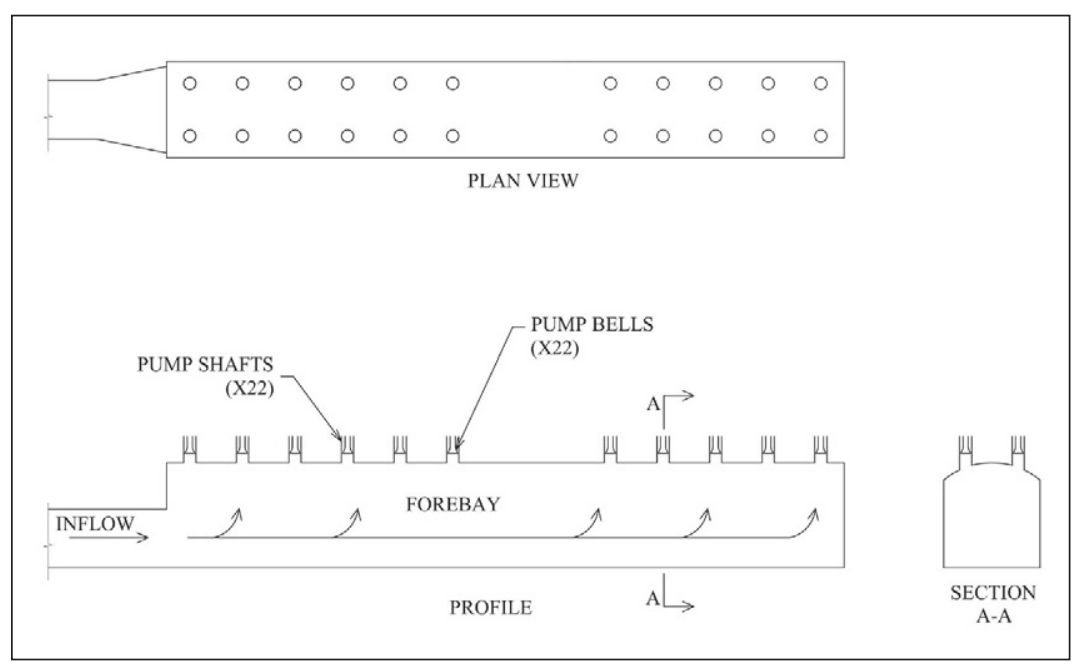

Figure 1. Simple schematic of the IPS3 forebay model.

be of particular concern. The problems may be compounded as header velocities increase and as the distance from the pump bell to the header $\left(Y_{\text {Bell }}\right)$ decreases. The previously identified problems can reduce pump efficiency, increase maintenance and, over time, cause damage to the pump. Because of this, the American National Standards Institute (ANSI) and the Hydraulic Institute (HI) have established acceptance criteria for physical model tests of open-bottom canned pump systems. These criteria include having a circulation angle, $\theta$, less than $5^{\circ}$; time-averaged velocities at points in the throat of the bell should be within 10\% of the cross-sectional area average velocity; and time-varying fluctuations at a point should produce a standard deviation from the time-averaged signal of less than $10 \%$. The circulation angle is defined as:

$$
\theta=\arctan \left(V_{t} / V_{A}\right)
$$

where $V_{t}$ is the average tangential velocity of the tip of the rotometer impeller, and $V_{A}$ is the average vertical velocity at the pump throat.

\section{HI guidelines}

In order to achieve acceptable hydraulic performance in open-bottom canned pump intakes, the $\mathrm{HI}$ has published the following design and operation 
guidelines for the benefit of the engineer and pump user.

1. A vortex suppressor is necessary to break up abnormal flow patterns ahead of the pump suction bell.

2. The maximum horizontal header velocity is $1.8 \mathrm{~m} / \mathrm{s}(6.0 \mathrm{ft} / \mathrm{s})$.

3. The maximum riser velocity is $1.5 \mathrm{~m} / \mathrm{s}$ $(5.0 \mathrm{ft} / \mathrm{s})$.

4. The minimum distance from the horizontal header to the bottom of the pump bell is $3.0 \times D$, where $D$ is the inside diameter of the riser.

5. The pump must hang centred in the vertical riser pipe.

6. The minimum distance from the submerged pump bell to the water surface is $1.0 \times D$.

7. Open-bottom canned intakes with flow rates exceeding 315 litres/s (5000 US gallons/min) per pump require a model test.

In researching this topic, any background or supporting information available was exhausted, but very little useful information was found. Therefore, data from physical models took on great significance in this study.

\section{Physical modelling}

As a basis for research, the data from three physical model studies were used. The first was that of an underground chamber with a horseshoe-shaped cross-section, known as Intake Pumping Station No. 3 (IPS3), from which water would be pumped to a water treatment plant and then to a nearby city. In the IPS3 physical model study, a pump forebay (header) using 11 pairs of identical pump shafts (risers) connected to the top of the forebay was modelled. Figure 1 shows a schematic of this model.

The second model study used was that of Intake Pumping Station No. 1 (IPS1). The setup of IPS1 was similar in design to that of IPS3, but it had only 10 pairs of pump shafts (risers), and the forebay (header) had a much smaller cross-sectional area and, consequently, a much higher header velocity than IPS3. A schematic of the IPS1 forebay model is shown in Figure 2.

The third model study included a single acrylic pipe (riser) that branched perpendicularly off a 61.0-cm (24-inch) line (header). This model was used to determine the similarities between the IPS3 and IPS1 data.

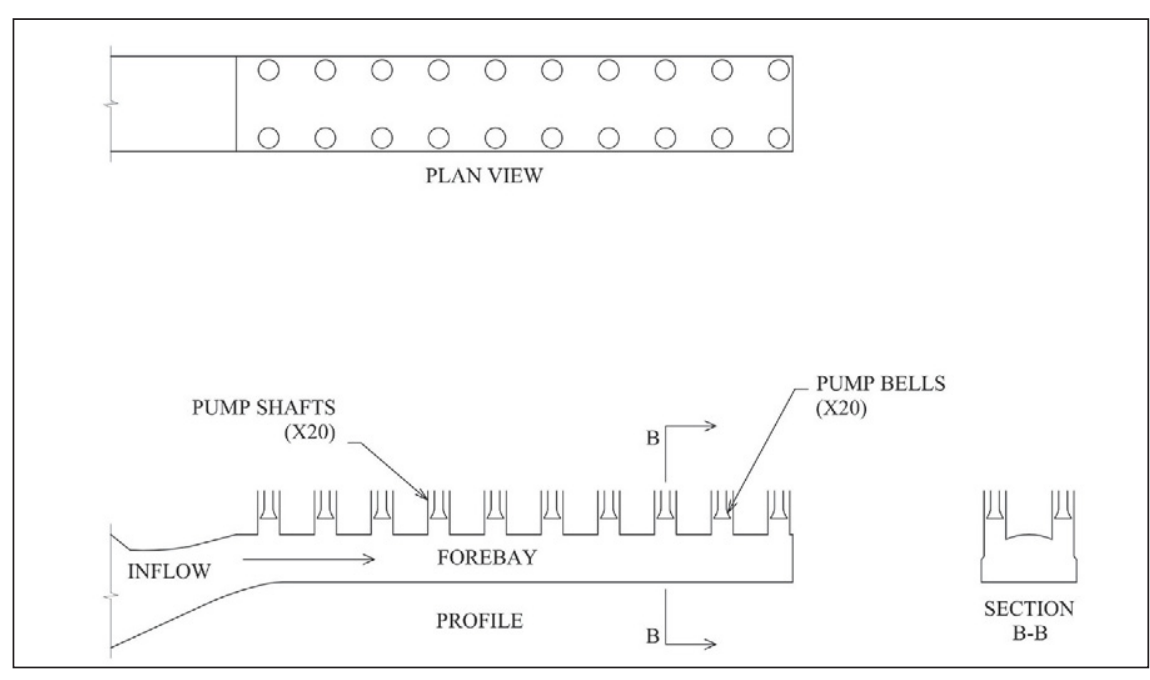

Figure 2. Simple schematic of IPS1.

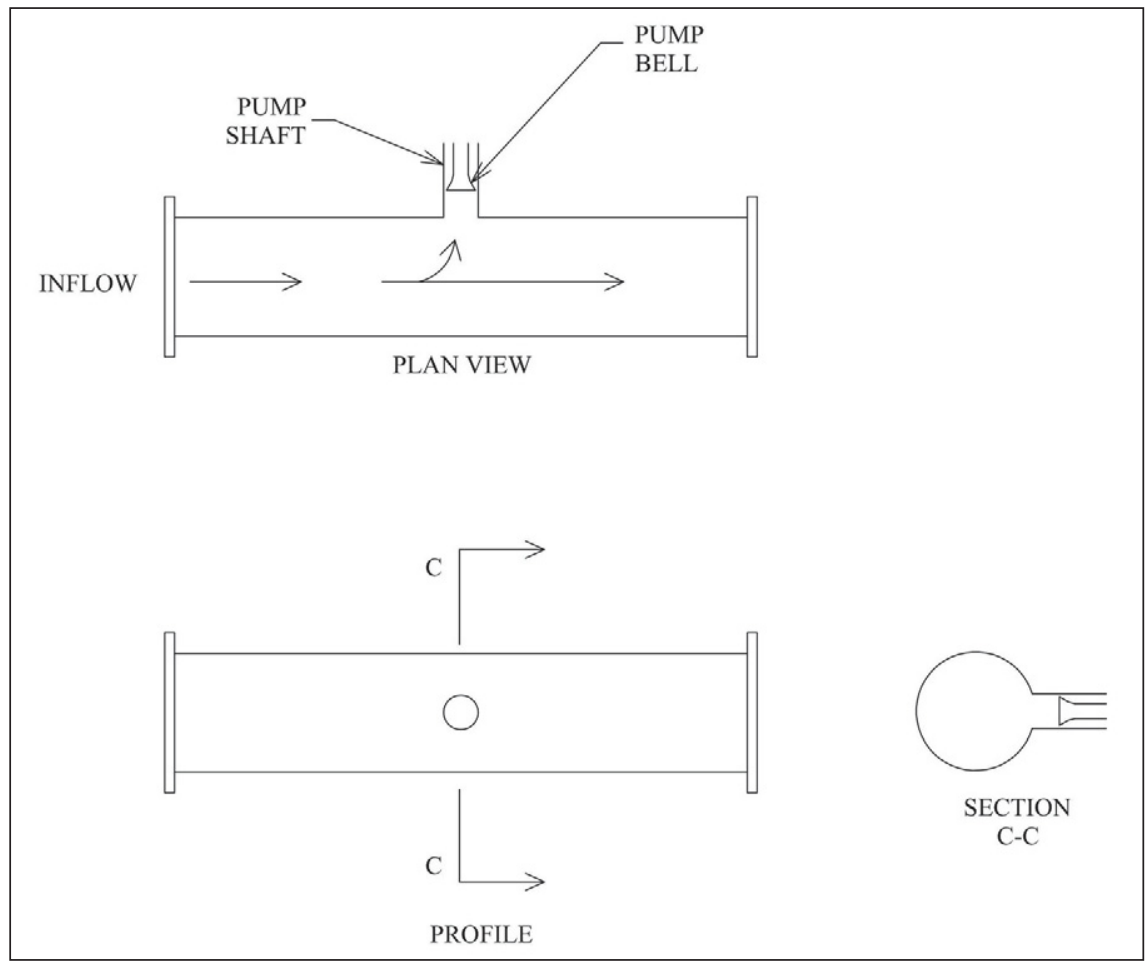

Figure 3. Schematic of the research model test setup.

Figure 3 shows a schematic of this research model.

The objective of this study was to determine system configurations that fall outside the HI guidelines. This makes it possible to install pumps closer to the header or operate the system at higher header or riser velocities than the $\mathrm{HI}$ guidelines suggest.

\section{The models}

The pump shafts for the models were constructed of clear acrylic tubing, which enabled the observation of the flow conditions to the pump bells, including vortex formation and persistence as well as other flow rotation. The pump throats and pump bells were also constructed of acrylic, using moulds to form the bells. The pump bells in each of the three model studies were tested with and without vortex suppressors. Figure 4 shows an example of the vortex suppressors used in these model studies.

Acrylic turn columns were fabricated and connected to the pump bells, allowing the bells to be rotated within the risers a full $360^{\circ}$. These turn columns allowed for the collection of velocity data at any position along the circumference of the pump throat using a single permanently mounted Pitot tube and static pressure tap. These instruments were used to evaluate the potential for velocity fluctuation and flow rotation problems at each pump throat. Differential 


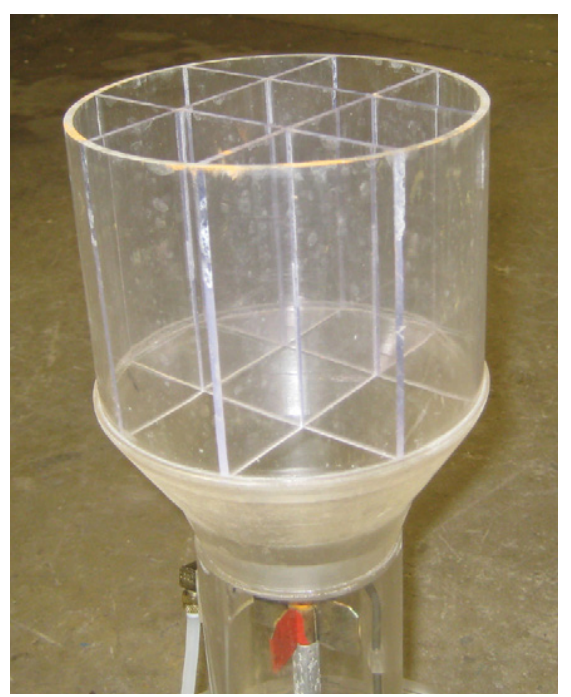

Figure 4. Typical vortex suppressor used in each of the three model studies.

pressure transducers, which are accurate to $1.0 \%$, were connected to each Pitot tube to measure the velocity head. A rotometer, as described in the HI Standard, was mounted in each pump throat. These rotometers were used to detect rotational flow entering the pump throat, enabling the calculation of the circulation angle.

Flow rates through the models were measured using calibrated orifice flow meters capable of reading to within $0.5 \%$. For low flow rates, a weigh tank was used to increase the accuracy in the flow measurement and flows were regulated using control valves.

\section{Conditions investigated}

With three variables in this study $\left(V_{r}, V_{h}\right.$ and $\left.Y_{B e l l}\right)$, the ratio of $V_{r} / V_{h}$ was used to create a dimensionless control parameter that would facilitate the comparison of different test scenarios. In this ratio, $V_{r}$ is the flow velocity through the riser, calculated immediately upstream of the bell in the shaft, and $V_{h}$ is the flow velocity through the header, calculated immediately upstream of the riser. This velocity ratio was used in comparing the hydraulic conditions at varying $Y_{\text {Bell }}$ distances. The tests run on each model had a $V_{r} / V_{h}$ ratio range of nearly one order of magnitude. The research model was tested with $V_{r} / V_{h}$ ratios ranging from 0.15 to 1.23 . In the IPS1 model study, the ratios ranged from 0.58 to 5.8 , while in the IPS3 model study, the $V_{r} / V_{h}$ ratios ranged from 3.5 to 34.8 .

While it would be desirable to operate each model over a wide flow range, physical limitations restricted the range of possible velocity ratios. Therefore, while $V_{r} / V_{h}$ ratios in the IPS3 model reached 34.8, Figures 5-7 only show ratios up to 8.0 because the IPS1 model and the research model were physically limited to ratios lower than 8.0. All IPS3 tests with ratios higher than 8.0 yielded results within the HI Standard.

\section{Test results}

Figure 5 ( $a$ and $b$ ) shows the velocity fluctuation and Figure 6 the velocity distribution, while Figure 7 compares the circulation angle calculated for each test condition. All these figures also illustrate the published $\mathrm{HI}$ standard in reference to the actual test data for comparison. The figure icons differentiate between specific model tests and describe the distance from the pump bell (in riser diameters) to the header $(\# x D)$.

As illustrated in Figures 5 and 6, the velocity ratio had a direct effect on the velocity fluctuations and the mean velocity distributions, with or without the vortex suppressor. The apparent uniform trend of the data for the velocity distributions and the velocity fluctuations both with and without the vortex suppressor is noteworthy. It appears that at higher velocity ratios, the distance from the pump bell to the header $\left(Y_{\text {Bell }}\right)$ has a less significant impact on the system. The circulation angle (Figure 7) was reduced by the addition of the vortex suppressor.
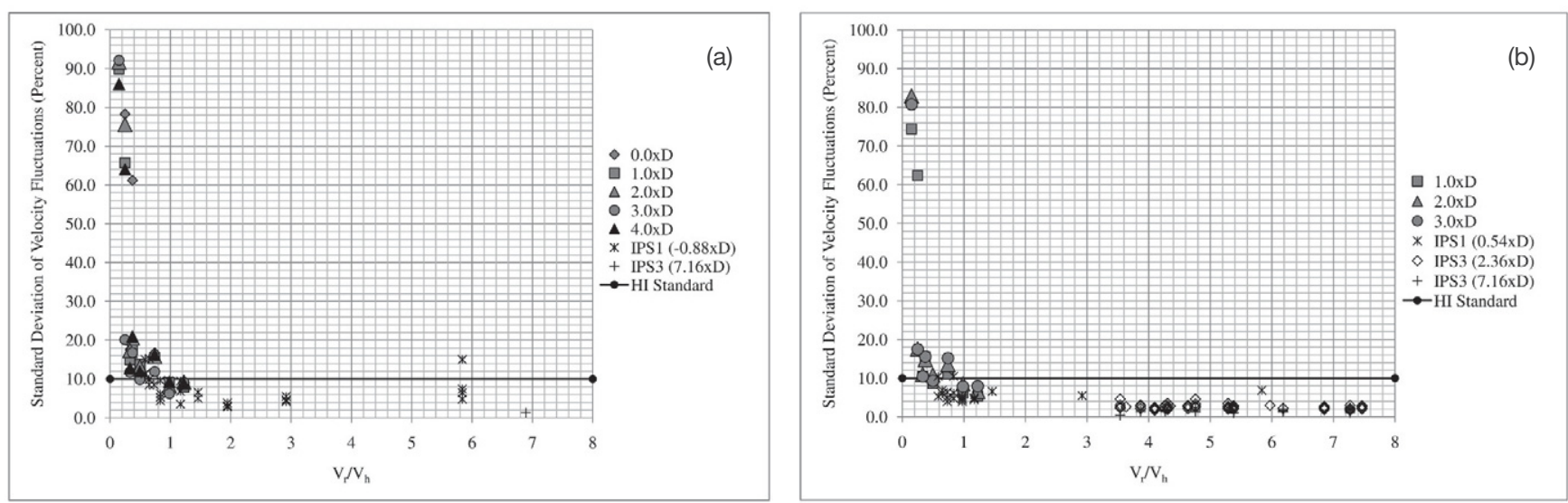

Figure 5. Standard deviation of the velocity fluctuations measured (a) without a vortex suppressor, and (b) with a vortex suppressor.
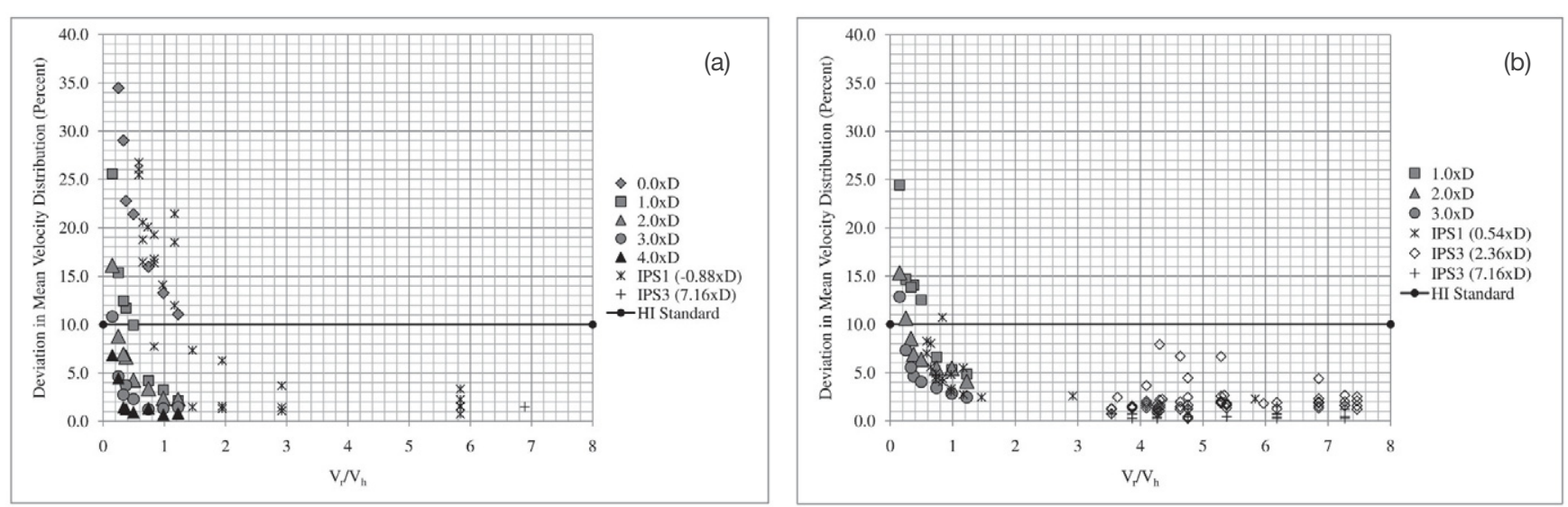

Figure 6. Deviation in the mean velocity distribution measured (a) without a vortex suppressor, and (b) with a vortex suppressor. 

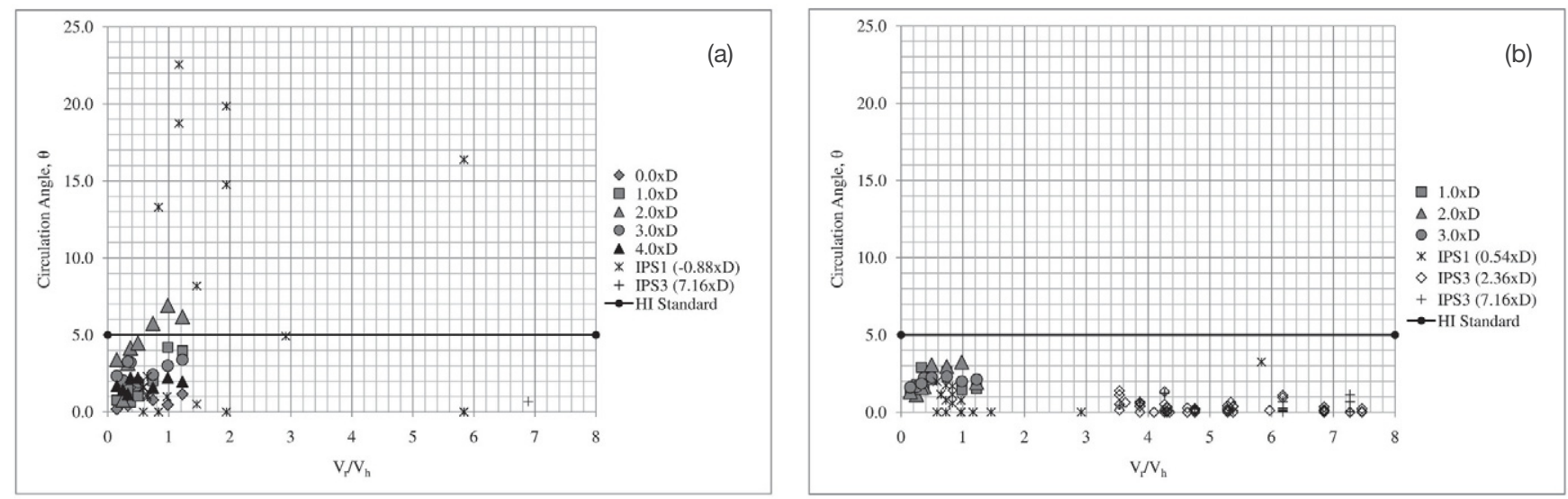

Figure 7. Circulation angle measured (a) without a vortex suppressor, and (b) with a vortex suppressor.

\section{Discussion}

\section{Velocity ratio}

While the HI's guidelines are an excellent resource for helping the engineer and pump user design and operate a system that falls within their acceptance criteria for model tests, the velocity ratio used in this research study would be an additional helpful tool that would allow engineers and pump users to change the configuration of the system to meet their needs. This could include having $Y_{\text {Bell }}$ distances less than the distance suggested by the $\mathrm{Hl}$, if need be, to access more water. It could also permit the use of higher average velocities in the riser if more water were needed, as long as the correct header velocity was used to achieve an acceptable velocity ratio.

With the use of $V_{r} / V_{h}$ ratios, the $H$ design parameters are extended beyond what is presently available. The determination can be made that without a vortex suppressor, at a $Y_{\text {Bell }}$ distance of 1.0xD, $V_{r} / V_{h}$ ratios above the 'minimum acceptance ratio' of 1.0 should result in conditions that would fall within the HI Standard. Therefore, systems that need to operate at a header velocity of $1.8 \mathrm{~m} / \mathrm{s}(6 \mathrm{ft} / \mathrm{s})$ - the $\mathrm{HI}$ limit - would need to keep the riser velocity above $1.8 \mathrm{~m} / \mathrm{s}$ ( $6 \mathrm{ft} / \mathrm{s})$. Conversely, if systems need to operate at a riser velocity of $1.5 \mathrm{~m} / \mathrm{s}(5 \mathrm{ft} / \mathrm{s})$ - the $\mathrm{HI}$ limit - the header velocity would need to stay below $1.5 \mathrm{~m} / \mathrm{s}(5 \mathrm{ft} / \mathrm{s})$.

\section{Vortex suppressor use}

The use of a vortex suppressor in these pumping conditions lowers the 'minimum acceptance ratio' to 0.63 . Therefore, if systems need to operate at the $1.8 \mathrm{~m} / \mathrm{s}$ header velocity limit with a vortex suppressor the riser velocity would need to exceed $1.2 \mathrm{~m} / \mathrm{s}$. For a riser velocity of $1.5 \mathrm{~m} / \mathrm{s}$, the header velocity would need to stay below $2.4 \mathrm{~m} / \mathrm{s}$. It should be remembered that one purpose of the vortex suppressor is to reduce vortices, and it may therefore need to be used in conditions where vortices are more likely. Again, these ratios must be used with consideration of the system's capacity limits in mind. These calculations offer much more freedom to the design engineer and pump user in the event that higher velocities than those given in the $\mathrm{HI}$ design parameters are required within the pump system.

The circulation angle was lowered by the vortex suppressor. If surface or sub-surface vortices were present in the tests, the vortex suppressor would have played an even more significant role.

It should be remembered that one of the stipulations in the HI Standard is that the design and operation guidelines given are especially applicable to pumps that will be pumping 315.5 litres/s (5000 USgpm) or less. The standard suggests that open-bottom canned intakes with flows greater than 315.5 litres/s per pump require a model test.

\section{Conclusions}

Open-bottom canned pumping systems are subject to a number of potential problems, as outlined in the introduction, which has prompted the $\mathrm{HI}$ to develop acceptance criteria for pump intakes. To help meet these criteria, the Institute has also established design guidelines for open-bottom canned pumps. These guidelines include the use of a vortex suppressor, maximum horizontal header velocity of $1.8 \mathrm{~m} / \mathrm{s}$, maximum riser velocity of $1.5 \mathrm{~m} / \mathrm{s}$, and minimum $Y_{\text {Bell }}$ of $3.0 \times D$, as detailed earlier.

The results from the three separate model studies discussed here indicate that the $V_{r} / V_{h}$ velocity ratio plays a very important role in determining the ability of a pump system to operate within the $\mathrm{HI}$ acceptance criteria. These results will be helpful in predicting pump system performance, based on this ratio and $Y_{\text {Bell}}$. With these results, the engineer and pump user will be able to position the pump bell in an open-bottom canned pump system below the suggested 3.0xD using an acceptable velocity ratio. With $Y_{\text {Bell }}$ distances greater than or equal to $1.0 \times D$, a velocity ratio of 1.0 is the minimum acceptable ratio for a pump system. Lowering the pump bell past 1.0xD would require raising the minimum acceptable velocity ratio to 1.4. Furthermore, engineers and pump users will also be able to use $V_{r} / V_{h}$ ratios to achieve the needed velocity through the header or riser without turbulence or rotation problems in the pump. A vortex suppressor is recommended for use in these pump systems.

It is apparent from the data that as the $V_{r} / V_{h}$ ratio increases, the velocity fluctuations and turbulence within the pump bell decrease. This implies that the magnitude of this ratio is the factor that plays the most significant role in velocity fluctuation and flow rotation within open-bottom canned vertical pump intake systems, although the ratio is limited by the capacity of the system itself. The $Y_{\text {Bell }}$ distance plays a significant but secondary role in this, giving less stable results as the distance decreases between the pump bell and the header.

\section{References}

For references, please contact David Sanders.

Contacts
David Sanders
Sunrise Engineering, Inc, Civil Energy Dept
12227 South Business Park Drive, Suite 220
Draper, UT 84020, USA.
Tel: +1 8015230100
Fax: +1 8015230990
E-mail: dsanders@sunrise-eng.com
www.sunrise-eng.com
Steven L. Barfuss
Utah Water Research Laboratory, Dept of Civil Engineering
Utah State University
Logan, UT 84322-8200, USA
E-mail: Barfuss@engineering.usu.edu
Michael C. Johnson
Utah Water Research Laboratory (as above)
E-mail: mikejnsn@engineering.usu.edu

